\title{
Efficient Placement of Distributed On-Chip Decoupling Capacitors in Nanoscale ICs
}

\author{
Mikhail Popovich, Eby G. Friedman \\ Department of Electrical and Computer Engineering \\ University of Rochester \\ Rochester, New York 14627 \\ [nhlover, friedman]@ece.rochester.edu
}

\author{
Radu M. Secareanu, Olin L. Hartin \\ MMSTL \\ Freescale Semiconductor \\ Tempe, Arizona 85284 \\ [r54143, lee.hartin]@freescale.com
}

\begin{abstract}
Decoupling capacitors are widely used to reduce power supply noise. On-chip decoupling capacitors have traditionally been allocated into the white space available on the die based on an unsystematic or ad hoc approach. In this way, large decoupling capacitors are often placed at a significant distance from the current load, compromising the signal integrity of the system. This issue of power delivery cannot be alleviated by simply increasing the size of the on-chip decoupling capacitors. To be effective, the on-chip decoupling capacitors should be placed physically close to the current loads. The area occupied by the onchip decoupling capacitor, however, is directly proportional to the magnitude of the capacitor. The minimum impedance between the on-chip decoupling capacitor and the current load is therefore fundamentally affected by the magnitude of the capacitor.

A distributed on-chip decoupling capacitor network is proposed in this paper. A system of distributed on-chip decoupling capacitors is shown to provide an efficient solution for providing the required on-chip decoupling capacitance under existing technology constraints. In a system of distributed onchip decoupling capacitors, each capacitor is sized based on the parasitic impedance of the power distribution grid. Various tradeoffs in a system of distributed on-chip decoupling capacitors are also discussed. Related simulation results for typical values of on-chip parasitic resistance are also presented. An analytic solution is shown to provide accurate distributed system. The worst case error is $0.003 \%$ as compared to SPICE. Techniques presented in this paper are applicable not only for current technologies, but also provide an efficient placement of the onchip decoupling capacitors in future technology generations.

Index Terms-Power distribution systems, power distribution grids, decoupling capacitors, power noise
\end{abstract}

\section{INTRODUCTION}

Decoupling capacitors are widely used to manage power supply noise [1]. Decoupling capacitors are an effective way to reduce the impedance of power delivery systems operating at high frequencies [2], [3]. A decoupling capacitor acts as a local reservoir of charge, which is released when the power supply voltage at a particular current load drops below some

This research is supported in part by the Semiconductor Research Corporation under Contract No. 2004-TJ-1207, the National Science Foundation under Contract Nos. CCR-0304574 and CCF-0541206, grants from the New York State Office of Science, Technology \& Academic Research to the Center for Advanced Technology in Electronic Imaging Systems, and by grants from Intel Corporation, Eastman Kodak Company, Intrinsix Corporation, and Freescale Semiconductor Corporation. tolerable level. Since the inductance scales slowly [4], the location of the decoupling capacitors significantly affects the design of power/ground $(\mathrm{P} / \mathrm{G})$ networks in high performance integrated circuits (ICs) such as microprocessors. At higher frequencies, a distributed system of decoupling capacitors will need to be placed on-chip to effectively manage the power supply noise [5].

The efficacy of decoupling capacitors depends upon the impedance of the conductors connecting the capacitors to the current loads and power sources. As described in [6], a maximum parasitic impedance between the decoupling capacitor and the current load (or power source) exists at which the decoupling capacitor is effective. Alternatively, to be effective, an on-chip decoupling capacitor should be placed such that both the power supply and the current load are located inside the appropriate effective radius [6]. The efficient placement of on-chip decoupling capacitors in nanoscale ICs is the subject of this paper. Unlike the methodology for placing a single lumped on-chip decoupling capacitor presented in [6], a system of distributed on-chip decoupling capacitors is proposed. A design methodology to estimate the parameters of the distributed system of on-chip decoupling capacitors is also presented, permitting allocation of the required on-chip decoupling capacitance under existing technology constraints.

The paper is organized as follows. The problem of placing on-chip decoupling capacitors in nanoscale ICs under technology constraints is formulated in Section II. The design of an on-chip distributed decoupling capacitor network is presented in Section III. Various design tradeoffs are discussed in Section IV. Related simulation results for typical values of the on-chip parasitic resistance are discussed in Section V. Some specific conclusions are summarized in Section VI.

\section{Placing On-Chip Decoupling Capacitors IN NANOSCALE ICS}

Decoupling capacitors have traditionally been allocated into the white space (those areas not occupied by the circuit elements) available on the die based on an unsystematic or ad hoc approach [7], [8], as shown in Fig. 1. In this way, decoupling capacitors are often placed at a significant distance from the current load. Conventional approaches for placing onchip decoupling capacitors result in oversized capacitors. The 
conventional allocation strategy, therefore, results in increased power noise, compromising the signal integrity of an entire system, as illustrated in Fig. 2. This issue of power delivery cannot be alleviated by simply increasing the size of the onchip decoupling capacitors. Furthermore, increasing the size of more distant on-chip decoupling capacitors results in wasted area, increased power, reduced reliability, and higher costs. A new design methodology is therefore required to account for technology trends in nanoscale ICs, such as increasing frequencies, larger die sizes, higher current demands, and reduced noise margins.

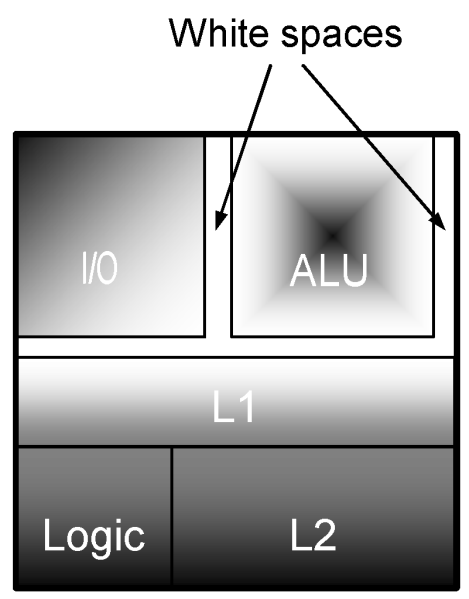

Fig. 1. Placement of on-chip decoupling capacitors using a conventional approach. Decoupling capacitors are allocated into the white space (those areas not occupied by the circuits elements) available on the die using an unsystematic or ad hoc approach. As a result, the power supply voltage drops below the minimum tolerable level for remote blocks (shown in dark grey). Low noise regions are light grey.

To be effective, a decoupling capacitor should be placed physically close to the current load. This requirement is naturally satisfied in board and package applications, since large capacitors are much smaller than the dimensions of the circuit board (or package) [9]. In this case, a lumped model of the decoupling capacitor provides sufficient accuracy [10].

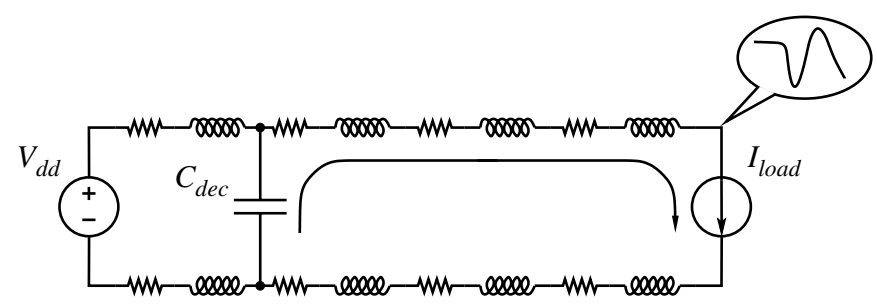

Fig. 2. A conventional on-chip decoupling capacitor. Typically, a large decoupling capacitor is placed at a greater distance from the current load due to physical limitations. Current flowing through the long power/ground lines results in large voltage fluctuations across the terminals of the current load

The size of an on-chip decoupling capacitor, however, is directly proportional to the area occupied by the capacitor and can require a significant portion of the on-chip area. The minimum impedance between an on-chip capacitor and the current load is fundamentally affected by the magnitude (and therefore the area) of the capacitor. Systematically partitioning the decoupling capacitor solves this issue. A system of on-chip distributed decoupling capacitors is illustrated in Fig. 3.

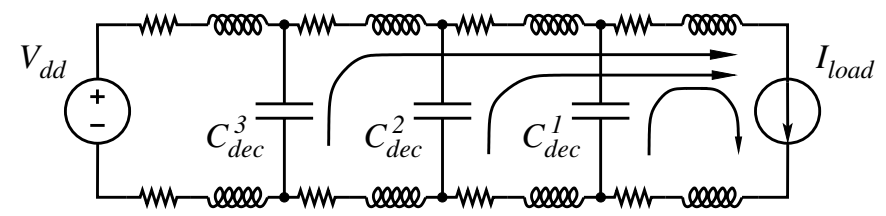

Fig. 3. A network of distributed on-chip decoupling capacitors. The magnitude of the decoupling capacitors is based on the impedance of the interconnect segment connecting a specific capacitor to a current load. Each decoupling capacitor is designed to only provide charge during a specific time interval.

In a system of distributed on-chip decoupling capacitors, each decoupling capacitor is sized based on the impedance of the interconnect segment connecting a capacitor to a current load. A particular capacitor only provides charge to a current load during a short period. The rationale behind the proposed scheme can be explained as follows. The capacitor closest to the current load is engaged immediately after the switching cycle is initiated. Once the first capacitor is depleted, the next capacitor is activated, providing a large portion of the total current drawn by the load. This procedure is repeated until the last capacitor becomes active. Similar to the hierarchical placement of decoupling capacitors presented in [3], [11], the proposed technique provides an efficient solution for providing the required on-chip decoupling capacitance based on specified capacitance density constraints. A system of distributed onchip decoupling capacitors should therefore be utilized to provide a low impedance, cost effective power delivery network in nanoscale ICs.

\section{DESIGN OF AN ON-CHIP DISTRIBUTED DECOUPLING CAPACITOR NETWORK}

As described in Section II, a system of distributed on-chip decoupling capacitors is an efficient solution for providing the required on-chip decoupling capacitance based on the maximum capacitance density available in a particular technology. A physical model of the proposed technique is illustrated in Fig. 4. For simplicity, two decoupling capacitors are assumed to provide the required charge drawn by the current load. Note that as the capacitor is placed farther from the current load, the magnitude of an on-chip decoupling capacitor increases due to relaxed constraints. In the general case, the proposed methodology can be extended to any practical number of onchip decoupling capacitors. Note that $Z_{1}$ is typically limited by a specific technology (determined by the impedance of a single metal wire) and the magnitude of $C_{1}$ (the area available in the vicinity of a circuit block).

A circuit model of the proposed system of distributed onchip decoupling capacitors is shown in Fig. 5. The impedance of the metal lines connecting the capacitors to the current 


$$
\begin{aligned}
\left.V_{C_{1}}\right|_{t=t_{r}} & =\frac{1}{2\left(C_{1}+C_{2}\right)^{3} t_{r}}\left[2 C_{1}^{3} t_{r}+C_{1}^{2} t_{r}\left(6 C_{2}-I_{\text {max }} t_{r}\right)-C_{2}^{2} t_{r}\left(2 C_{2}\left(I_{\text {max }} R_{2}-1\right)\right.\right. \\
& \left.\left.+I_{\text {max }} t_{r}\right)+2 C_{1} C_{2}\left(C_{2}^{2}\left(1-e^{-\frac{\left(C_{1}+C_{2}\right) t_{r}}{C_{1} C_{2} R_{2}}}\right) I_{\max } R_{2}^{2}+C_{2}\left(3-I_{\max } R_{2}\right) t_{r}-I_{\max } t_{r}^{2}\right)\right], \\
\left.V_{C_{2}}\right|_{t=t_{r}} & =\frac{1}{2\left(C_{1}+C_{2}\right)^{3} t_{r}}\left[2 C_{1}^{3} t_{r}+C_{2}^{2} t_{r}\left(2 C_{2}-I_{\max } t_{r}\right)+2 C_{1} C_{2} t_{r}\left(C_{2}\left(3+I_{\text {max }} R_{2}\right)\right.\right. \\
& \left.\left.-I_{\text {max }} t_{r}\right)+C_{1}^{2}\left(2 C_{2}^{2}\left(e^{-\frac{\left(C_{1}+C_{2}\right) t_{r}}{C_{1} C_{2} R_{2}}}-1\right) I_{\max } R_{2}^{2}+2 C_{2}\left(3+I_{\text {max }} R_{2}\right) t_{r}-I_{\text {max }} t_{r}^{2}\right)\right] .
\end{aligned}
$$

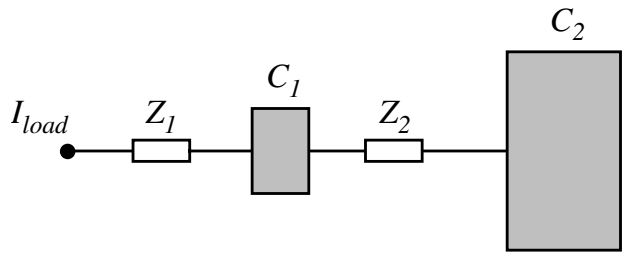

Fig. 4. A physical model of the proposed system of distributed on-chip decoupling capacitors. Two capacitors are assumed to provide the required charge drawn by the load. $Z_{1}$ and $Z_{2}$ denote the impedance of the metal lines connecting $C_{1}$ to the current load and $C_{2}$ to $C_{1}$, respectively.

load is modeled as resistors $R_{1}$ and $R_{2}$. Modeling the power and ground paths as simple resistors is a tradeoff, providing sufficient accuracy to estimate the required on-chip decoupling capacitance, while dramatically reducing the model complexity. A triangular current source is assumed to characterize the current load. The magnitude of the current source increases linearly, reaching the maximum current $I_{\max }$ at rise time $t_{r}$, i.e., $I_{\text {load }}(t)=I_{\max } \frac{t}{t_{r}}$. The maximum tolerable ripple at the current load is $10 \%$ of the power supply voltage.

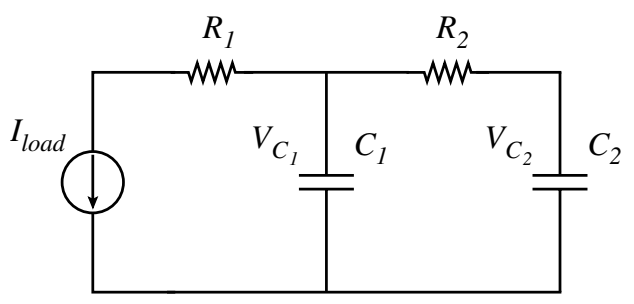

Fig. 5. A circuit model of an on-chip distributed decoupling capacitor network. The impedance of the metal lines is modeled as $R_{1}$ and $R_{2}$, respectively.

Note from Fig. 5 that since the charge drawn by the current load is provided by the on-chip decoupling capacitors, the voltage across the capacitors during discharge drops below the initial power supply voltage. The required charge during the entire switching event is thus determined by the voltage drop across $C_{1}$ and $C_{2}$.

The voltage across the decoupling capacitors at the end of the switching cycle $\left(t=t_{r}\right)$ can be determined from Kirchhoff's laws [12]. Writing KVL and KCL for each of the loops (see Fig. 5), the system of differential equations for the voltage across $C_{1}$ and $C_{2}$ at $t_{r}$ is

$$
\begin{gathered}
\frac{d V_{C_{1}}}{d t}=\frac{V_{C_{2}}-V_{C_{1}}}{R_{2} C_{1}}-\frac{I_{\text {load }}}{C_{1}}, \\
\frac{d V_{C_{2}}}{d t}=\frac{V_{C_{1}}-V_{C_{2}}}{R_{2} C_{2}} .
\end{gathered}
$$

Simultaneously solving (1) and (2) and applying the initial conditions, the voltage across $C_{1}$ and $C_{2}$ at the end of the switching activity is determined by (3) and (4), respectively. $I_{\max }$ is the maximum magnitude of the current load and $t_{r}$ is the rise time.

Note that the voltage across $C_{1}$ and $C_{2}$ after discharge is determined by the magnitude of the decoupling capacitors and the parasitic resistance of the metal line(s) between the capacitors. The voltage across $C_{1}$ after the switching cycle, however, depends upon the resistance of the $\mathrm{P} / \mathrm{G}$ paths connecting $C_{1}$ to a current load and is

$$
V_{C_{1}}=V_{\text {load }}+I_{\max } R_{1},
$$

where $V_{\text {load }}$ is the voltage across the terminals of a current load. Assuming $V_{\text {load }} \geq 0.9 V_{d d}$ and $V_{C_{1}}^{\max }=V_{d d}$ (meaning that $C_{1}$ is infinitely large), the upper bound for $R_{1}$ is

$$
R_{1}^{\max }=\frac{V_{d d}(1-\alpha)}{I_{\max }}
$$

where $\alpha$ is the ratio of the minimum tolerable voltage across the terminals of a current load to the power supply voltage ( $\alpha=0.9$ in this paper). If $R_{1}>R_{1}^{\max }$, no solution exists for providing sufficient charge drawn by the load. In this case, the circuit block should be partitioned, reducing the current demands $\left(I_{\max }\right)$.

Note that expressions for determining the voltage across the decoupling capacitors are transcendental functions. No closed-form solution, therefore, exists. From (3) and (4), the design space can be graphically obtained for determining the maximum tolerable resistance $R_{2}$ and the minimum magnitude of the capacitors, maintaining the voltage across the load equal to or greater than the minimum allowable level. The voltage across $C_{1}$ after discharge as a function of $C_{1}$ and $R_{2}$ is depicted in Fig. 6.

Observe from Fig. 6 that the voltage across capacitor $C_{1}$ increases exponentially with capacitance, saturating for large $C_{1}$. The voltage across $C_{1}$, however, is almost independent 


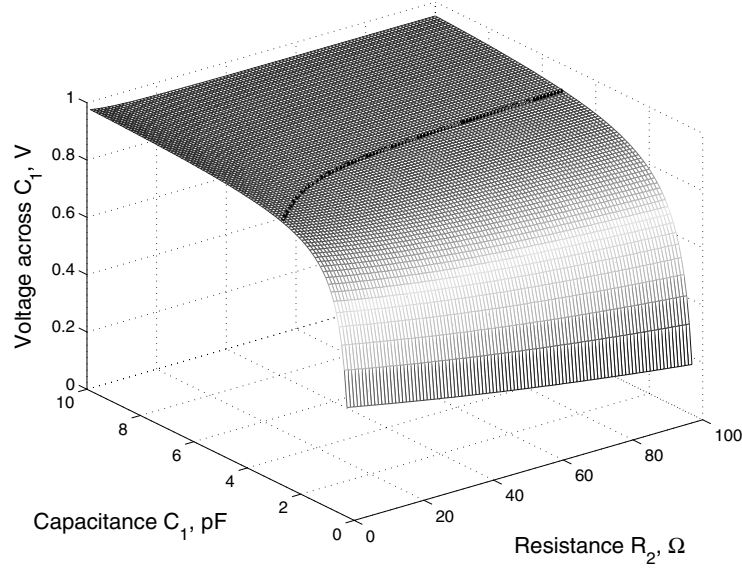

(a)

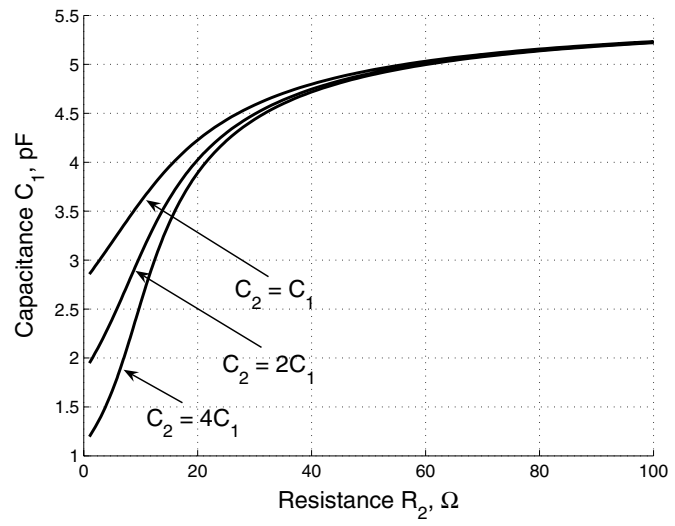

(b)

Fig. 6. Voltage across $C_{1}$ during discharge as a function of $C_{1}$ and $R_{2}$ : $I_{\max }=0.01 \mathrm{~mA}, V_{d d}=1$ volt, and $t_{r}=100 \mathrm{ps}$. a) Assuming $C_{1}=$ $C_{2}$ and $R_{1}=10 \Omega$, the minimum tolerable voltage across $C_{1}$, resulting in $V_{\text {load }} \geq 0.9 V_{d d}$, is 0.91 volts (shown as a black equipotential line). b) The design space for determining $C_{1}$ and $R_{2}$ resulting in the voltage across $C_{1}$ equal to 0.91 volts.

of $R_{2}$, decreasing slightly with $R_{2}$ (see Fig. 6(a)). This behavior can be explained as follows. As a current load draws charge from the decoupling capacitors, the voltage across the capacitors drops below the initial level. The charge released by a capacitor is proportional to the capacitance and the voltage drop. A larger capacitance therefore results in a smaller voltage drop. From Fig. 5, note that as resistance $R_{2}$ increases, capacitor $C_{2}$ becomes less effective (a larger portion of the total current is provided by $C_{1}$ ). As a result, the magnitude of $C_{1}$ is increased to maintain the voltage across the load above the minimum tolerable level. Similarly, a larger $C_{2}$ results in a smaller $C_{1}$. As $C_{2}$ is increased, a larger portion of the total current is provided by $C_{2}$, reducing the magnitude of $C_{1}$. This phenomenon is well pronounced for small $R_{2}$, diminishing with larger $R_{2}$, as illustrated in Fig. 6(b).

In general, to determine the parameters of the system of distributed on-chip decoupling capacitors, the following assumptions are made. The parasitic resistance of the metal line(s) connecting capacitor $C_{1}$ to the current load is known. $R_{1}$ is determined by technology constraints (the sheet resistance) and by design constraints (the maximum available metal resources). The minimum voltage level at the load is $V_{\text {load }}=0.9 V_{d d}$. The maximum magnitude of the current load $I_{\text {max }}$ is $0.01 \mathrm{~A}$, the rise time $t_{r}$ is $100 \mathrm{ps}$, and the power supply voltage $V_{d d}$ is one volt. Note that the voltage across $C_{2}$ after discharge as determined by (4) is also considered a design parameter. Since the capacitor $C_{2}$ is connected directly to the power supply (a shared power rail), the voltage drop across $C_{2}$ appears on the global power line, compromising the signal integrity of the overall system. The voltage across $C_{2}$ at $t_{r}$ is therefore based on the maximum tolerable voltage fluctuations on the P/G line during discharge (the voltage across $C_{2}$ at the end of the switching cycle is set to 0.95 volts).

The system of equations to determine the parameters of an on-chip distributed decoupling capacitor network as depicted in Fig. 5 is

$$
\begin{aligned}
V_{\text {load }} & =V_{C_{1}}-I_{\max } R_{1}, \\
V_{C_{1}} & =f\left(C_{1}, C_{2}, R_{2}\right), \\
V_{C_{2}} & =f\left(C_{1}, C_{2}, R_{2}\right), \\
\frac{I_{\max } t_{r}}{2} & =C_{1}\left(V_{d d}-V_{C_{1}}\right)+C_{2}\left(V_{d d}-V_{C_{2}}\right),
\end{aligned}
$$

where $V_{C_{1}}$ and $V_{C_{2}}$ are the voltage across $C_{1}$ and $C_{2}$ and determined by (3) and (4), respectively. Equation (10) states that the total charge drawn by the current load is provided by $C_{1}$ and $C_{2}$. Note that in the general case with the current load determined a priori, the total charge is the integral of $I_{\text {load }}(t)$ from zero to $t_{r}$. Solving (7) for $V_{C_{1}}$ and substituting into (8), $C_{1}, C_{2}$, and $R_{2}$ are determined from (8) to (10), as discussed in the following section.

\section{Design Tradeoffs in a Distributed ON-ChIP DECOUPLING CAPACITOR NETWORK}

To design a system of distributed on-chip decoupling capacitors, the parasitic resistances and capacitances should be determined based on design and technology constraints. As shown in Section III, in a system composed of two decoupling capacitors (see Fig. 5) with known $R_{1}, R_{2}, C_{1}$, and $C_{2}$ are determined from the system of equations, (7)-(10). Note that since this system of equations involves transcendental functions, a closed-form solution cannot be determined. To determine the system parameters, the system of equations, (7)(10), is solved numerically [13].

Various tradeoff scenarios are discussed in this section. The dependence of the system parameters on $R_{1}$ is presented in Section IV-A. The design of a distributed on-chip decoupling capacitor network with minimum magnitude $C_{1}$ is discussed in Section IV-B. 


\section{A. Dependence of System Parameters on $R_{1}$}

The parameters of a distributed on-chip decoupling capacitor network for typical values of $R_{1}$ are listed in Table I. Note that the minimum magnitude of $R_{2}$ exists for which the parameters of the system can be determined. If $R_{2}$ is sufficiently small, the distributed decoupling capacitor network degenerates to a system with a single capacitor (where $C_{1}$ and $C_{2}$ are merged). For the parameters listed in Table I, the minimum magnitude of $R_{2}$ is four ohms, as determined from numerical simulations.

TABLE I

DEPENDENCE OF THE PARAMETERS OF THE DISTRIBUTED ON-CHIP DECOUPLING CAPACITOR NETWORK ON $R_{1}$

\begin{tabular}{c||c|c|c|c}
\hline \hline \multirow{2}{*}{$\begin{array}{c}R_{1} \\
(\Omega)\end{array}$} & \multicolumn{2}{c|}{$R_{2}=5(\Omega)$} & \multicolumn{2}{c}{$R_{2}=10(\Omega)$} \\
\cline { 2 - 5 } & $C_{1}$ & $C_{2}$ & $C_{1}$ & $C_{2}$ \\
& $(\mathrm{pF})$ & $(\mathrm{pF})$ & $(\mathrm{pF})$ & $(\mathrm{pF})$ \\
\hline \hline 1 & 1.35 & 7.57 & 3.64 & 3.44 \\
2 & 2.81 & 5.50 & 4.63 & 2.60 \\
3 & 4.54 & 3.64 & 5.88 & 1.77 \\
4 & 6.78 & 1.87 & 7.56 & 0.92 \\
5 & 10.00 & 0 & 10.00 & 0 \\
\hline \hline & \multicolumn{3}{c}{$V_{\text {dd }}=1 \mathrm{~V}, V_{\text {load }}=0.9 \mathrm{~V}}$, \\
$t_{r}=100 \mathrm{ps}$, and $I_{\text {max }}=0.01 \mathrm{~A}$ \\
\hline \hline
\end{tabular}

Note that the parameters of a distributed on-chip decoupling capacitor network are determined by the parasitic resistance of the P/G line(s) connecting $C_{1}$ to the current load. As $R_{1}$ increases, the capacitor $C_{1}$ increases substantially (see Table I). This increase in $C_{1}$ is due to $R_{1}$ becoming comparable to $R_{2}$, and $C_{1}$ providing a greater portion of the total current. Alternatively, the system of distributed on-chip decoupling capacitors degenerates to a single oversized capacitor. The system of distributed on-chip decoupling capacitors should therefore be carefully designed. Since the distributed on-chip decoupling capacitor network is strongly dependent upon the first level of interconnection $\left(R_{1}\right), C_{1}$ should be placed as physically close as possible to the current load, reducing $R_{1}$. If such an allocation is not practically possible, the current load should be partitioned, permitting an efficient allocation of the distributed on-chip decoupling capacitors under specific technology constraints.

\section{B. Minimum $C_{1}$}

In practical applications, the size of $C_{1}$ (the capacitor closest to the current load) is typically limited by technology constraints, such as the maximum capacitance density, and design constraints, such as the available area. The magnitude of the first capacitor in a distributed system is therefore typically small. In this section, the dependence of the distributed onchip decoupling capacitor network on $R_{1}$ is determined for minimum $C_{1}$. A target magnitude of $1 \mathrm{pF}$ is assumed for $C_{1}$. The parameters of a system of distributed on-chip decoupling capacitors as a function of $R_{1}$ under the constraint of a minimum $C_{1}$ are listed in Table II. Note that $V_{C_{2}}$ denotes the voltage across $C_{2}$ after discharge.
TABLE II

DISTRIBUTED ON-CHIP DECOUPLING CAPACITOR NETWORK AS A FUNCTION OF $R_{1}$ UNDER THE CONSTRAINT OF A MINIMUM $C_{1}$

\begin{tabular}{c||c|c|c|c|c|c}
\hline \hline \multicolumn{1}{c||}{$\begin{array}{c}R_{1} \\
(\Omega)\end{array}$} & $R_{2}(\Omega)$ & $C_{2}(\mathrm{pF})$ & $R_{2}(\Omega)$ & $C_{2}(\mathrm{pF})$ & $R_{2}(\Omega)$ & $C_{2}(\mathrm{pF})$ \\
\hline \hline 1 & 2 & 5.59 & 5 & 8.69 & 4.68 & 8.20 \\
2 & 2 & 6.68 & 5 & 11.64 & 3.46 & 8.40 \\
3 & 2 & 8.19 & 5 & 17.22 & 2.28 & 8.60 \\
4 & 2 & 10.46 & 5 & 31.70 & 1.13 & 8.80 \\
5 & 2 & 14.21 & 5 & 162.10 & - & - \\
\hline \hline & \multicolumn{6}{c}{$V_{\text {dd }}=1 \mathrm{~V}, V_{\text {load }}=0.9 \mathrm{~V}, t_{r}=100 \mathrm{ps}}$, \\
\hline
\end{tabular}

Note that two scenarios are considered in Table II to evaluate the dependence of a distributed system of on-chip decoupling capacitors. In the first scenario, the distributed onchip decoupling capacitor network is designed to maintain the minimum tolerable voltage across the terminals of a current load. In this case, the magnitude of $C_{2}$ increases with $R_{1}$, becoming impractically large for large $R_{2}$. In the second scenario, an additional constraint (the voltage across $C_{2}$ ) is applied to reduce the voltage fluctuations on the shared $P / G$ lines. In this case, as $R_{1}$ increases, $C_{2}$ slightly increases. In order to satisfy the constraint for $V_{C_{2}}, R_{2}$ should be significantly reduced for large values of $R_{1}$, meaning that the second capacitor should be placed close to the first capacitor. As $R_{1}$ is further increased, $R_{2}$ becomes negligible, implying that capacitors $C_{1}$ and $C_{2}$ should be merged to provide the required charge to the distant current load.

\section{CAse Study}

The dependence of the system of distributed on-chip decoupling capacitors on the current load and the parasitic impedance of the power delivery system is described in this section to quantitatively illustrate the previously presented concepts. The load is modeled as a ramp current source with a $100 \mathrm{ps}$ rise time. The minimum tolerable voltage across the load terminals is $90 \%$ of the power supply level. The magnitude of the on-chip decoupling capacitors for various parasitic resistances of the metal lines connecting the capacitors to the current load is listed in Table III. The parameters of the distributed on-chip decoupling capacitor network listed in Table III are determined for two amplitudes of the current load.

The parameters of the system of distributed on-chip decoupling capacitors are analytically determined from (7)(10). The resulting power supply noise is estimated using SPICE and compared to the maximum tolerable level (the minimum voltage across the load terminals $\left.V_{\text {load }}^{\min }\right)$. The maximum voltage drop across $C_{2}$ at the end of the switching activity is also estimated and compared to $V_{C_{2}}^{\text {min }}$. Note that the analytic solution produces accurate estimates of the onchip decoupling capacitors for typical parasitic resistances of a power distribution grid. The maximum error in this case study is $0.003 \%$. 
TABLE III

THE MAGNITUDE OF ON-CHIP DECOUPLING CAPACITORS AS A FUNCTION OF THE PARASITIC RESISTANCE OF THE POWER/GROUND LINES CONNECTING THE CAPACITORS TO THE CURRENT LOAD

\begin{tabular}{|c|c|c|c|c|c|c|c|c|c|c|}
\hline \multirow{2}{*}{$\begin{array}{l}R_{1} \\
(\Omega)\end{array}$} & \multirow{2}{*}{$\begin{array}{l}R_{2} \\
(\Omega)\end{array}$} & \multirow{2}{*}{$\begin{array}{c}I_{\max } \\
\text { (A) }\end{array}$} & \multirow{2}{*}{$\begin{array}{c}C_{1} \\
(\mathrm{pF})\end{array}$} & \multirow{2}{*}{$\begin{array}{c}C_{2} \\
(\mathrm{pF})\end{array}$} & \multicolumn{2}{|c|}{$V_{\text {load }}(\mathrm{mV})$} & \multirow{2}{*}{$\begin{array}{l}\text { Error } \\
(\%)\end{array}$} & \multicolumn{2}{|c|}{$V_{C_{2}}(\mathrm{mV})$} & \multirow{2}{*}{$\begin{array}{c}\text { Error } \\
(\%)\end{array}$} \\
\hline & & & & & $V_{\text {load }}^{\min }$ & SPICE & & $V_{C_{2}}^{\min }$ & SPICE & \\
\hline 0.5 & 4.5 & 0.01 & 0 & 9.99999 & 900 & 899.999 & 0.0001 & 950 & 949.999 & 0.0001 \\
\hline 0.5 & 6 & 0.01 & 1.59747 & 6.96215 & 900 & 899.986 & 0.002 & 950 & 949.983 & 0.002 \\
\hline 0.5 & 8 & 0.01 & 2.64645 & 4.97091 & 900 & 899.995 & 0.0006 & 950 & 949.993 & 0.0004 \\
\hline 0.5 & 10 & 0.01 & 3.22455 & 3.87297 & 900 & 899.997 & 0.0003 & 950 & 949.996 & 0.0004 \\
\hline 0.5 & 12 & 0.01 & 3.59188 & 3.17521 & 900 & 899.998 & 0.0002 & 950 & 949.997 & 0.0003 \\
\hline 0.5 & 14 & 0.01 & 3.84641 & 2.69168 & 900 & 899.998 & 0.0002 & 950 & 949.997 & 0.0003 \\
\hline 0.5 & 16 & 0.01 & 4.03337 & 2.33650 & 900 & 899.999 & 0.0001 & 950 & 949.998 & 0.0002 \\
\hline 0.5 & 18 & 0.01 & 4.17658 & 2.06440 & 900 & 899.998 & 0.0002 & 950 & 949.998 & 0.0002 \\
\hline 0.5 & 20 & 0.01 & 4.28984 & 1.84922 & 900 & 899.999 & 0.0001 & 950 & 949.998 & 0.0002 \\
\hline 0.5 & 1.5 & 0.025 & 0 & 24.99930 & 900 & 899.998 & 0.0002 & 950 & 949.998 & 0.0002 \\
\hline 0.5 & 2 & 0.025 & 4.25092 & 17.56070 & 900 & 899.999 & 0.0001 & 950 & 949.999 & 0.0001 \\
\hline 0.5 & 3 & 0.025 & 7.97609 & 11.04180 & 900 & 899.999 & 0.0001 & 950 & 949.999 & 0.0001 \\
\hline 0.5 & 4 & 0.025 & 9.67473 & 8.06921 & 900 & 899.999 & 0.0001 & 950 & 949.999 & 0.0001 \\
\hline 0.5 & 5 & 0.025 & 10.65000 & 6.36246 & 900 & 899.999 & 0.0001 & 950 & 949.999 & 0.0001 \\
\hline 0.5 & 6 & 0.025 & 11.2838 & 5.25330 & 900 & 899.999 & 0.0001 & 950 & 949.999 & 0.0001 \\
\hline 0.5 & 7 & 0.025 & 11.72910 & 4.47412 & 900 & 899.999 & 0.0001 & 950 & 949.999 & 0.0001 \\
\hline 0.5 & 8 & 0.025 & 12.05910 & 3.89653 & 900 & 899.999 & 0.0001 & 950 & 949.999 & 0.0001 \\
\hline 0.5 & 9 & 0.025 & 12.31110 & 3.44905 & 900 & 899.980 & 0.002 & 950 & 949.973 & 0.003 \\
\hline \multicolumn{11}{|c|}{$V_{d d}=1 \mathrm{~V}$, and $t_{r}=100 \mathrm{ps}$} \\
\hline
\end{tabular}

From Table III, note that in the case of a large $R_{2}$, the distributed decoupling capacitor network degenerates to a system with a single capacitor. Capacitor $C_{1}$ is therefore excessively large. Conversely, if $C_{2}$ is placed close to $C_{1}$ ( $R_{2}$ is small), $C_{2}$ is excessively large and the system behaves as a single capacitor. An optimal ratio $\frac{R_{2}}{R_{1}}$ therefore exists for specific characteristics of the current load that results in the minimum budgeted on-chip decoupling capacitance. Alternatively, in this case, both capacitors provide an equal portion of the total charge (see Table III for $R_{1}=0.5 \Omega$ and $R_{2}=10 \Omega$ ).

\section{CONCLUSIONS}

A system of distributed on-chip decoupling capacitors has been proposed in this paper. A distributed on-chip decoupling capacitor network is an efficient solution for providing the required on-chip decoupling capacitance under existing technology constraints. In a system of distributed on-chip decoupling capacitors, each capacitor is sized based on the parasitic impedance of the power delivery system. Initially, the capacitor closest to the current load provides the required charge. Once the first capacitor is depleted, the next decoupling capacitor is activated, providing a large portion of the total current drawn by the load. This procedure is repeated until the last decoupling capacitor becomes active. Hierarchically allocating the on-chip decoupling capacitors greatly relaxes the technology constraints for physically distant capacitors, making the distant on-chip decoupling capacitors more effective.

\section{REFERENCES}

[1] M. Popovich, A. V. Mezhiba, and E. G. Friedman, Power Distribution Networks with On-Chip Decoupling Capacitors, Springer Publishers, 2008 (in press).
[2] M. Popovich and E. G. Friedman, "Impedance Characteristics of Decoupling Capacitors in Multi-Power Distribution Systems," Proceedings of the IEEE International Conference on Electronics, Circuits and Systems, pp. 160-163, December 2004.

[3] M. Popovich and E. G. Friedman, "Decoupling Capacitors for MultiVoltage Power Distribution Systems," IEEE Transactions on Very Large Scale Integration (VLSI) Systems, Vol. 14, No. 3, pp. 217-228, March 2006.

[4] A. V. Mezhiba and E. G. Friedman, "Scaling Trends of On-Chip Power Distribution Noise," IEEE Transactions on Very Large Scale Integration (VLSI) Systems, Vol. 12, No. 4, pp. 386-394, April 2004.

[5] M. Popovich and E. G. Friedman, "Noise Aware Decoupling Capacitors for Multi-Voltage Power Distribution Systems," Proceedings of the IEEE International Symposium of Quality Electronic Design, pp. 334-339, March 2005

[6] M. Popovich, E. G. Friedman, M. Sotman, A. Kolodny, and R. M. Secareanu, "Maximum Effective Distance of On-Chip Decoupling Capacitors in Power Distribution Grids," Proceedings of the ACM Great Lakes Symposium on VLSI, pp. 173-179, April/May 2006.

[7] H. Su, S. S. Sapatnekar, and S. R. Nassif, "Optimal Decoupling Capacitor Sizing and Placement for Standard-Cell Layout Designs," IEEE Transactions on Computer-Aided Design of Integrated Circuits and Systems, Vol. 22, No. 4, pp. 428-436, April 2003.

[8] S. Zhao, K. Roy, and C.-K. Koh, "Decoupling Capacitance Allocation and Its Application to Power-Supply Noise-Aware Floorplanning," IEEE Transactions on Computer-Aided Design of Integrated Circuits and Systems, Vol. 21, No. 1, pp. 81-92, January 2002.

[9] J. Kim, et al., "Separated Role of On-Chip and On-PCB Decoupling Capacitors for Reduction of Radiated Emission on Printed Circuit Board," Proceedings of the IEEE International Symposium on Electromagnetic Compatibility, pp. 531-536, August 2001.

[10] T. Hubing, "Effective Strategies for Choosing and Locating Printed Circuit Board Decoupling Capacitors," Proceedings of the IEEE International Symposium on Electromagnetic Compatibility, pp. 632-637, August 2005.

[11] L. D. Smith, R. E. Anderson, D. W. Forehand, T. J. Pelc, and T. Roy, "Power Distribution System Design Methodology and Capacitor Selection for Modern CMOS Technology," IEEE Transactions on Advanced Packaging, Vol. 22, No. 3, pp. 284-291, August 1999.

[12] M. E. Van Valkenburg, Network Analysis, Prentice-Hall, 1974.

[13] Mathematica 5.2, Wolfram Research, Inc. 